BNL-73535-2005-CP

\title{
Review of Non-neutron and Neutron Nuclear Data, 2004
}

\author{
Norman E. Holden
}

\author{
National Nuclear Data Center, Brookhaven National Laboratory, Upton, NY USA 11973-5000
}

\begin{abstract}
Review articles are in preparation for the 2004 edition of the CRC Handbook of Chemistry and Physics dealing with the evaluation of both non-neutron and neutron nuclear data. Data on the discovery of element 110 , Darmstadtium, and element 111 have been officially accepted, while data on element 118 have been withdrawn. Data to be presented include revised values for very short-lived nuclides, long-lived nuclides and beta-beta decay measurements. There has been a reassessment of the spontaneous fission (sf) half-lives, which distinguishes between sf decay half-lives and cluster decay half-lives and with cluster-fission decay. New measurements of isotopic abundance values for many elements will be discussed with an emphasis on the minor isotopes of interest for use in neutron activation analysis measurements. Neutron resonance integrals will be discussed emphasizing the differences between the calculated values obtained from the analytical integration over neutron resonances and the measured values in a neutron reactor-spectrum, which does not quite conform to the assumed 1/E neutron energy spectrum. The method used to determine the neutron resonance integral from measurement, using neutron activation analysis, will be discussed.
\end{abstract}

\section{INTRODUCTION}

The published scientific literature is scanned and periodically evaluated for any new or revised nuclear data measurements. Pertinent information on isotopic masses, isotopic composition, radioactive half-lives, atomic weights, alpha particle, beta, proton, positron, isomeric transition energy and branching ratios, $\gamma-$ ray energies and intensities. Nuclear spin, parity, magnetic dipole and electric quadrupole moments and other nuclear information are reviewed and "best values" are recommended and published [1]. For neutron data, cross sections for thermal energy (room temperature) reactions, resonance integrals, scattering lengths and Maxwellian averaged cross sections at a neutron energy value of $30 \mathrm{keV}$ for astrophysical applications are reviewed and "best values" are recommended and published [2].

\section{ELEMENTS AND NUCLIDES}

Data reported for new elements to be placed on the Periodic Table of Chemical Elements are reviewed. It is determined which set of data is the first to have found that new element and whether or not there has been verification of that claim. One group is allowed to claim discovery and have the privilege of supplying the name of this new element. New claims of discovery for elements with $Z=113$ and $Z=115$ has appeared in the literature [3]. Data supporting a discovery of element $Z=118$ was withdrawn, when the data could not be confirmed. Another claim of discovery of element $Z=118$ has been made [4].

Two claims of discovery have been acknowledged, which resulted in officially naming element $Z=110$ Darmstadtium and its addition to the Periodic Table [5]. A second element $Z=111$, Roentgenium, is in the process of being officially approved later this year.

There have been a number of new short-lived nuclides discovered, which are located far from the line of beta stability. In the past eight years, there has been a $20 \%$ increase in the number of nuclides discovered. There have been 504 new nuclides added to bring the total number of nuclides to 3060 , which does not include electromagnetic isomers. The newly discovered nuclides have been found with $40 \%$ on the neutron deficient side of stability (lighter masses of an element) and $60 \%$ on the neutron excess side (heavier masses of that element). This ratio has not been systematic throughout the periodic table. For the light and medium weight elements up to $Z=75,2$ out of 3 new nuclides have been discovered on the neutron excess side of stability, while for heavier elements, $Z=76$ to $Z=100,3$ out of 4 new nuclides have been found on the neutron deficient side of stability. For many of these nuclides, some radiation- 
has been followed to allow an estimate of the radioactive half-life. In other cases, only the existence of the nuclide from its mass and charge can be inferred.

\section{ISOTOPIC COMPOSITION}

The isotopic composition of an element is determined by measuring the ratio of the individual stable nuclides of the element. The abundance values of the stable nuclides are normalized to unity. The number of significant digits in the major isotope(s) may require truncation in the number of significant digits (lost information) in the minor isotope. This minor isotope is often the one isotope of interest for neutron activation analysis purposes in determining the cross section or the resonance integral. If one uses the original isotope ratio measurements with the isotopic composition, one can usually obtain an extra significant digit for the minor isotope. In Table 1, the first value listed is the isotopic composition followed by the calculated value from isotope ratios. This latter abundance value has not been normalized to unity.

TABLE 1. Isotopic Abundances of Minor Isotopes

$$
\begin{aligned}
& \text { Isot. Comp. New ' Isot. Comp. New } \\
& \begin{array}{llllll}
{ }^{36} \mathrm{~S} & .01(1) & .0146(1) & { }^{40} \mathrm{~K} & .0117(1) & .01167(2)
\end{array} \\
& { }^{46} \mathrm{Ca} \quad .004(3) \quad .0035(10) \quad{ }^{78} \mathrm{Kr} \quad .355(3) \quad .3552(3) \\
& { }^{84} \mathrm{Sr} \quad .56(1) \quad .557(2) \quad{ }^{152} \mathrm{Gd} \quad .20(1) \quad .203(1) \\
& { }^{156} \text { Dy .056(3) } \quad .0549(1) \quad{ }^{158} \mathrm{Dy} \quad .095(3) \quad .0951(2) \\
& \quad{ }^{174} \mathrm{Hf} .16(1) \quad .162(4) \quad{ }^{190} \mathrm{Pt} \quad .014(1) \quad .0136(1) \\
& { }^{196} \mathrm{Hg} \quad .15(1) \quad .153(1)
\end{aligned}
$$

In recent years, many isotopic compositions of chemical elements have been revised to better fit the average properties of the elements. The philosophy is to examine the total range in nature of the abundance of each isotope and to position the recommended value as the midpoint of this total range, independent of the value for the most common source of the element. One result is the much larger uncertainty assigned to the mean value compared to previous values. Variations of isotopic abundances for selected elements have been reviewed recently [6]. Fortunately, most natural variations occur in light isotopes, where the neutron cross sections are usually relatively small.

\section{RADIOACTIVE HALF-LIVES}

In electrodynamics, symmetry principles guarantee the absence of a mass for the photon and the absolute conservation of the electric charge. In theories of particle physics, neutrinos and electrons (leptons) and protons and neutrons (baryons) have lepton numbers and baryon numbers of +1 , while their anti-particles have lepton numbers and baryon numbers of -1 . The "Standard Model" (SM) of particle physics explains the stability of the proton by the law of baryon number conservation. In modern theories, this law is not considered to be absolute because of the lack of an underlying symmetry principle. Extensions of the SM theory incorporate interactions that would violate baryon number conservation by assuming the symmetry associated with baryon number conservation is broken at a certain energy scale. Such interactions could lead to processes such as the decay of the proton, where the baryon number would change from +1 to 0 . Proton stability has been searched for in various experiments. It has not yet been found and the upper limit [7] for the half-life of the proton can be seen in Table 2.

One of the upper limits in Table 2 is due to the search for charge non-conservation (CNC) reactions, a violation of charge conservation in ${ }^{71} \mathrm{Ga}$ decay. The normal $\beta$ decay process in ${ }^{71} \mathrm{Ga}$ is energetically forbidden. In the case of $\mathrm{CNC}$, no electron would be emitted and its rest mass energy of $511 \mathrm{keV}$ would be available as extra decay energy. The CNC decay energy is positive by $+275 \mathrm{keV}$ (or energetically possible), while for charge conserving decay, the decay energy is negative, $-236 \mathrm{keV}$, (or energetically forbidden). If this decay process can be detected, it would mean that the electric charge is not conserved in this reaction. This would correspond to a violation of a basic law in physics that has long been assumed to be true. The ${ }^{71} \mathrm{Ga}$ solar neutrino experiments placed an upper limit of $3.5 \times 10^{26}$ years on this decay [8], so CNC has not yet been observed.

Double beta decay $(\beta \beta)$ is a rare transition between two nuclei with the same mass number that changes the nuclear charge number by two units. It is of interest as a tool for the study of lepton conservation and neutrino properties. Two electrons (and possibly two anti-neutrinos) must be emitted to conserve electric charge. Only $2 v \beta \beta$ decays are listed in Table 2. No 0v $\beta \beta$ decays are listed. Note also that ${ }^{209} \mathrm{Bi}$, which was thought to be a stable nuclide, actually decays by $\alpha$ particle emission [9]. 
Table 2. Radioactive Half-lives, Long-lived Nuclei

$$
\begin{aligned}
& \text { Isot. Decay Years Isot. Decay Years } \\
& { }^{1} \mathrm{H} \quad \text { proton }>35 \cdot 10^{27}{ }^{40} \mathrm{Ca} \text { ec-ec }>59 \cdot 10^{20} \\
& { }^{48} \mathrm{Ca} \quad \beta^{-} \beta^{-} \quad 43 \cdot 10^{18} \quad{ }^{50} \mathrm{Cr} \quad \beta^{+} \text {-ec }>13 \cdot 10^{17} \\
& { }^{58} \mathrm{Ni} \quad \beta^{+}-\mathrm{ec}>4 \cdot 10^{19} \quad{ }^{64} \mathrm{Zn} \cdot \text { ec-ec }>23 \cdot 10^{17} \\
& { }^{71} \mathrm{Ga} \quad \beta^{-} \quad>35 \cdot 10^{25} \quad{ }^{76} \mathrm{Ge} \beta^{-} \beta^{-} \quad 18 \cdot 10^{20} \\
& { }^{82} \mathrm{Se} \quad \beta^{-} \beta^{-} \quad 12 \cdot 10^{19} \quad{ }^{78} \mathrm{Kr} \quad \beta^{+} \beta^{+} \quad 20 \cdot 10^{20} \\
& { }^{94} \mathrm{Zr} \quad \beta^{-} \beta^{-}>1 \cdot 10^{17} \quad{ }^{96} \mathrm{Zr} \quad \beta^{-} \beta^{-} \quad 21 \cdot 10^{18} \\
& { }^{92} \text { Mo } \beta^{+}-\text {ec }>\cdot 19 \cdot 10^{18} \quad{ }^{100} \text { Mo } \beta^{-} \beta^{-} \quad 80 \cdot 10^{17} \\
& { }^{96} \mathrm{Ru} \quad \beta^{+} \beta^{+}>31 \cdot 10^{15}{ }^{106} \mathrm{Cd} \quad \beta^{+} \beta^{+}>24 \cdot 10^{19} \\
& { }^{108} \mathrm{Cd} \mathrm{ec-ec}>41 \cdot 10^{16}{ }^{114} \mathrm{Cd} \quad \beta^{-} \beta^{-}>6 \cdot 10^{17} \\
& { }^{116} \mathrm{Cd} \quad \beta^{-} \beta^{-} \quad 38 \cdot 10^{18}{ }^{124} \mathrm{Sn} \quad \beta^{-} \beta^{-}>22 \cdot 10^{17} \\
& { }^{128} \mathrm{Te} \quad \beta \beta^{-} \quad 2 \cdot 10^{24} \quad{ }^{130} \mathrm{Te} \quad \beta^{-} \beta^{-} \quad 8 \cdot 10^{20} \\
& { }^{124} \mathrm{Xe} \text { ec-ec }>1 \cdot 10^{17}{ }^{134} \mathrm{Xe} \quad \beta^{-} \beta^{-}>11 \cdot 10^{15} \\
& { }^{136} \mathrm{Xe} \beta^{-} \beta^{-}>8 \cdot 10^{20}{ }^{130} \mathrm{Ba} \text { ec-ec } 22 \cdot 10^{20} \\
& { }^{132} \mathrm{Ba} \text { ec-ec } 13 \cdot 10^{20} \quad{ }^{136} \mathrm{Ce} \quad \beta^{+} \beta^{+}>18 \cdot 10^{15} \\
& { }^{138} \mathrm{Ce} \text { ec-ec }>9 \cdot 10^{13}{ }^{150} \mathrm{Nd} \quad \beta^{-} \beta^{-} \quad 7 \cdot 10^{18} \\
& { }^{160} \mathrm{Gd} \quad \beta^{-} \beta^{-}>19 \cdot 10^{18}{ }^{176} \mathrm{Yb} \quad \beta^{-} \beta^{-}>16 \cdot 10^{16} \\
& { }^{180} \mathrm{~W} \text { ec-ec } \quad 7 \cdot 10^{16} \quad{ }^{186} \mathrm{~W} \quad \beta^{-} \beta^{-} \quad 37 \cdot 10^{17} \\
& { }^{209} \mathrm{Bi} \quad \alpha \quad 19 \cdot 10^{18} \quad{ }^{238} \mathrm{U} \quad \beta^{-} \beta^{-} \quad 20 \cdot 10^{20}
\end{aligned}
$$

\section{Other Nuclear Information}

For heavy elements, decay modes include spontaneous fission (sf) decay [10] and cluster decay [11]. In sf decay, the nucleus breaks up into two approximately equal reaction products, both of which have very large masses ( 15 to 40 times an $\alpha$ particle). In cluster decay, the emitted particle has a smaller mass (about 3 to 8 times an $\alpha$ particle). Using the sf and total half-life values, the sf branching ratio (percentage of the time that a nucleus decays by sf mode) has been determined [12] for heavy nuclides. These sf branching ratios are listed in Table 3.

For heavy chemical elements, a path to the super heavy elements is being explored. Among new elements being discovered, element 113 and 115 were mentioned earlier.
Table 3. Spontaneous Fission Branching Ratios

$$
\begin{aligned}
& \text { Isot. Percent Isot. Percent Isot. Percent } \\
& { }^{232} \mathrm{Th} \quad 12 \cdot 10^{-10} \quad{ }^{234} \mathrm{U} \quad 16 \cdot 10^{-10} \quad{ }^{235} \mathrm{U} \quad 70 \cdot 10^{-10} \\
& { }^{236} \mathrm{U} \quad 94 \cdot 10^{-6} \quad{ }^{238} \mathrm{U} \quad 54 \cdot 10^{-6} \quad{ }^{236} \mathrm{Pu} \quad 19 \cdot 10^{-8} \\
& { }^{238} \mathrm{Pu} \quad 18 \cdot 10^{-8} \quad{ }^{239} \mathrm{Pu} \quad 3 \cdot 10^{-12} \quad{ }^{240} \mathrm{Pu} \quad 57 \cdot 10^{-7} \\
& { }^{241} \mathrm{Pu} \quad 24 \cdot 10^{-15} \quad{ }^{242} \mathrm{Pu} \quad 56 \cdot 10^{-5} \quad{ }^{244} \mathrm{Pu} \quad 0.12 \\
& { }^{241} \mathrm{Am} \quad 36 \cdot 10^{-11} \quad{ }^{243} \mathrm{Am} \quad 37 \cdot 10^{-10} \quad{ }^{240} \mathrm{Cm} \quad 39 \cdot 10^{-7} \\
& { }^{242} \mathrm{Cm} \quad 64 \cdot 10^{-7} \quad{ }^{243} \mathrm{Cm} \quad 52 \cdot 10^{-10} \quad{ }^{244} \mathrm{Cm} \quad 14 \cdot 10^{-5} \\
& { }^{245} \mathrm{Cm} \quad 61 \cdot 10^{-8} \quad{ }^{246} \mathrm{Cm} \quad 0.026 \quad{ }^{248} \mathrm{Cm} \quad 0.082 \\
& { }^{250} \mathrm{Cm} \quad 86 . \quad{ }^{249} \mathrm{Bk} \quad 49 \cdot 10^{-9} \quad{ }^{237} \mathrm{Cf} \quad 10 . \\
& { }^{238} \mathrm{Cf} \quad 100 . \quad{ }^{240} \mathrm{Cf} \quad \sim 2 \quad{ }^{246} \mathrm{Cf} \quad 0.00023 \\
& { }^{248} \mathrm{Cf} \quad 0.0029 \quad{ }^{249} \mathrm{Cf} \quad 44 \cdot 10^{-8} \quad{ }^{250} \mathrm{Cf} \quad 0.077 \\
& { }^{252} \mathrm{Cf} \quad 3.08 \quad{ }^{254} \mathrm{Cf} \quad 99.3 \quad{ }^{256} \mathrm{Cf} \quad 100 . \\
& { }^{253} \mathrm{Es} \quad 89 \cdot 10^{-7}{ }^{255} \mathrm{Es} \quad 0.0042 \quad{ }^{244} \mathrm{Fm} \quad 100 . \\
& { }^{246} \mathrm{Fm} \quad 8 . \quad{ }^{248} \mathrm{Fm} \quad 0.10 \quad{ }^{250} \mathrm{Fm} \quad 0.007 \\
& { }^{252} \mathrm{Fm} \quad 0.0023 \quad{ }^{254} \mathrm{Fm} \quad 0.059 \quad{ }^{255} \mathrm{Fm} \quad 24 \cdot 10^{-6} \\
& { }^{256} \mathrm{Fm} \quad 91.9 \quad{ }^{257} \mathrm{Fm} \quad 0.21 \quad{ }^{258} \mathrm{Fm} \quad 100 . \\
& { }^{259} \mathrm{Fm} \quad 100 . \quad{ }^{260} \mathrm{Fm} \quad 100 . \quad{ }^{245} \mathrm{Md} \quad 100 . \\
& { }^{247} \mathrm{Md} \quad 100 . \quad{ }^{259} \mathrm{Md} \sim 100 . \quad{ }^{250} \text { No } \sim 100 \\
& { }^{252} \text { No } 32 . \quad{ }^{254} \text { No } 0.17 \quad{ }^{256} \text { No } 0.53 \\
& { }^{258} \text { No } 100 . \quad{ }^{260} \text { No } 100 . \quad{ }^{262} \text { No } 100 . \\
& { }^{253} \mathrm{Lr} \quad 1.3 \quad{ }^{259} \mathrm{Lr} \quad 20 . \quad{ }^{261} \mathrm{Lr} \quad 100 . \\
& { }^{153} \mathrm{Rf} \sim 100 . \quad{ }^{255} \mathrm{Rf} \quad \text { 52. } \quad{ }^{256} \mathrm{Rf} \quad 99.7 \\
& { }^{258} \mathrm{Rf} \quad 87 . \quad{ }^{259} \mathrm{Rf} \quad \text { 8. } \quad{ }^{260} \mathrm{Rf} \quad 100 . \\
& { }^{255} \mathrm{Db} \quad \sim 20 . \quad{ }^{260} \mathrm{Db} \quad 9.6 \quad{ }^{263} \mathrm{Db} \quad 55 . \\
& { }^{258} \mathrm{Sg} \quad 100 . \quad{ }^{260} \mathrm{Sg} \quad 50 . \quad{ }^{264} \mathrm{Hs} \quad 50 .
\end{aligned}
$$

The data for the initial discovery of element 118 was withdrawn when no Laboratory, even the originating $\mathrm{Lab}$ could confirm the original data. Since then another claim has been made by another Laboratory. Table 4 lists the data on nuclides with $Z>109$, where superscript " $\mathrm{g}$ " refers to the ground state and the superscript " $m$ " refers to an excited energy state. 
Table 4. Nuclear Data on Very Heavy Elements

\begin{tabular}{|c|c|c|c|}
\hline Nuclide & Half-life & Mode & Energy \\
\hline${ }^{267} \mathrm{Ds}$ & $\sim 3 \mu s$ & $\alpha$ decay & $11.6 \mathrm{Mev}$ \\
\hline${ }^{269} \mathrm{Ds}$ & $0.17 \mathrm{~ms}$ & $\alpha$ decay & $11.1 \mathrm{MeV}$ \\
\hline${ }^{270 m}$ Ds & $\sim 6 . \mathrm{ms}$ & $\alpha$ decay & $11.0-12.2 \mathrm{MeV}$ \\
\hline${ }^{270 g} \mathrm{Ds}$ & $\sim 10 . \mu \mathrm{s}$ & $\alpha$ decay & $11.0 \mathrm{MeV}$ \\
\hline${ }^{271 \mathrm{~m}} \mathrm{Ds}$ & $\sim 1.1 \mathrm{~ms}$ & $\alpha$ decay & $10.7 \mathrm{MeV}$ \\
\hline${ }^{2713} \mathrm{Ds}$ & $\sim 56 . \mathrm{ms}$ & $\alpha$ decay & $10.7 \mathrm{MeV}$ \\
\hline${ }^{273 m} \mathrm{Ds}$ & $0.76 \mathrm{~ms}$ & $\alpha$ decay & $11.8 \mathrm{MeV}$ \\
\hline${ }^{273 g} \mathrm{Ds}$ & 118. ms & $\alpha$ decay & $9.73 \mathrm{MeV}$ \\
\hline${ }^{279} \mathrm{Ds}$ & $\sim 0.3 \mathrm{~s}$ & spon. fiss. & \\
\hline${ }^{281} \mathrm{Ds}$ & $\sim 9.6 \mathrm{~s}$ & spon. fiss. & \\
\hline${ }^{272} \mathrm{Rg}$ & $1.5 \mathrm{~ms}$ & $\alpha$ decay & $11.0 \mathrm{MeV}$ \\
\hline${ }^{279} \mathrm{Rg}$ & $\sim 0.17 \mathrm{~s}$ & $\alpha$ decay & $10.4 \mathrm{MeV}$ \\
\hline${ }^{280} \mathrm{Rg}$ & $\sim 3.6 \mathrm{~s}$ & $\alpha$ decay & $9.8 \mathrm{MeV}$ \\
\hline${ }^{277} 112$ & $0.48 \mathrm{~ms}$ & $\alpha$ decay & $11.2-11.7 \mathrm{MeV}$ \\
\hline${ }^{282} 112$ & 1. $\mathrm{ms}$ & spon. fiss. & \\
\hline${ }^{283} 112$ & $\sim 6 . s$ & $\alpha$ decay & $9.5 \mathrm{MeV}$ \\
\hline${ }^{284} 112$ & $0.1 \mathrm{~s}$ & spon. fiss. & \\
\hline${ }^{285} 112$ & $\sim 34 . \mathrm{s}$ & $\alpha$ decay & $9.2 \mathrm{MeV}$ \\
\hline${ }^{283} 113$ & $\sim 0.1 \mathrm{~s}$ & $\alpha$ decay & $10.1 \mathrm{MeV}$ \\
\hline${ }^{284} 113$ & $\sim 0.5 \mathrm{~s}$ & $\alpha$ decay & $10.0 \mathrm{MeV}$ \\
\hline${ }^{286} 114$ & $0.3 \mathrm{~s}$ & $\alpha$ decay & $10.0 \mathrm{MeV}$ \\
\hline${ }^{287} 114$ & $\sim 1 . \mathrm{s}$ & $\alpha$ decay & $10.0 \mathrm{MeV}$ \\
\hline${ }^{288} 114$ & $\sim 0.6 \mathrm{~s}$ & $\alpha$ decay & $10.0 \mathrm{MeV}$ \\
\hline${ }^{289} 114$ & $\sim 2.7 \mathrm{~s}$ & $\alpha$ decay & $9.8 \mathrm{MeV}$ \\
\hline${ }^{287} 115$ & $\sim 0.03 \mathrm{~s}$ & $\alpha$ decay & $10.6 \mathrm{MeV}$ \\
\hline${ }^{288} 115$ & $\sim 87 . \mathrm{ms}$ & $\alpha$ decay & $10.5 \mathrm{MeV}$ \\
\hline${ }^{290} 116$ & $\sim 15 . \mathrm{ms}$ & $\alpha$ decay & $10.9 \mathrm{MeV}$ \\
\hline${ }^{291} 116$ & $\sim 6 . \mathrm{ms}$ & $\alpha$ decay & $10.7 \mathrm{MeV}$ \\
\hline${ }^{292} 116$ & $\sim 33 . \mathrm{ms}$ & $\alpha$ decay & $10.6 \mathrm{MeV}$ \\
\hline${ }^{293} 116$ & $\sim 0.05 \mathrm{~s}$ & $\alpha$ decay & $10.5 \mathrm{MeV}$ \\
\hline${ }^{294} 118$ & $\sim 1.8 \mathrm{~ms}$ & $\alpha$ decay & $11.8 \mathrm{MeV}$ \\
\hline
\end{tabular}

The neutron cross section is a measure of the probability of the interaction of a neutron with a nuclide. In thermal reactors, neutrons are produced in the fission process and are slowed down by collisions with moderator atoms. The neutron slowing down spectrum varies inversely with the energy, E. The resonance integral $(\mathrm{RI})$ is the probability of neutron reactions in this region, i.e., the integral of the cross section over the epithermal flux (assumed 1/E per unit energy interval). The flux is not proportional to $1 / \mathrm{E}$, but $\beta$ is introduced and is reactor dependent. Flux per unit energy interval is proportional to $1 / \mathrm{E}^{1+\beta}$ [13]. RI upper limits are important in threshold reactions. Integration to $20 \mathrm{MeV}$ distorts the RI [14].

\section{ACKNOWLEDGEMENT}

This research was supported in part by the United States Department of Energy (USDOE) under the contract DE-AC02-98CH10886.

\section{REFERENCES}

1. Holden, N. E., "Table of the Isotopes (Revised 2002)", Handbook of Chemistry and Physics $85^{\text {th }}$ edition, edited by D. R. Lide, Boca Raton, Fl: CRC Press Inc., 2004, section11, pp. 50-201.

2. Holden, N. E., "Neutron Scattering and Absorption Properties (Revised 2003)", Handbook of Chemistry and Physics, $85^{\text {th }}$ edition, edited by D. R. Lide, Boca Raton, FI: CRC Press, 2004, section 11, pp. 202-217.

3. Oganessian, Yu. Ts. et al., Phys. Rev. /C 69, 021601 (2004).

4. Oganessian, Yu. Ts. et al., JINR Report D7-2002-287, Dubna, Russia 2002 pp. 1-12.

5. Holden, N. E., and Coplen, T. B., Chemistry International 26, 8-9 (2004).

6. Coplen, T. B. et al., Pure Appl. Chem. 74, 1987-2017 (2002).

7. Zdesenko, Yu. G., and Tretyak, V. I., Phys. Lett. /B 553 135-140 (2003).

8. Norman, E. B., et al., Phys. Rev. ID 53 4086-4088 (1996).

9. de Marcillac, P. et al., Nature 422 876-878 (2003).

10. Holden, N. E., and Hoffman, D. C., Pure Appl. Chem. 72, 1525-1562 (2000).

11. Rose, H. J., and Jones, G. A., Nature 307 245-247 (1984).

12. Holden, N. E., "Spontaneous Fission Half-lives and Their Systematics" in Proc. Int. Conf. Phys. Of Nuclear Science \& Technology, LaGrange Park, IL: American Nuclear Society, 1998, pp. 663-670.

13. Ryves, T. B., Metrologia 5 119-124 (1969).

14. Holden, N. E., "A Warning on Fission Resonance Integrals-Caveat Utor", in Proc. Conf. Fifty Years with Nuclear Fission, LaGrange Park, IL: American Nuclear Society, 1989, pp. 946-948. 


\section{DISCLAIMER}

This report was prepared as an account of work sponsored by an agency of the United States Government. Neither the United States Government nor any agency thereof, nor any of their employees, nor any of their contractors, subcontractors, or their employees, makes any warranty, express or implied, or assumes any legal liability or responsibility for the accuracy, completeness, or any third party's use or the results of such use of any information, apparatus, product, or process disclosed, or represents that its use would not infringe privately owned rights. Reference herein to any specific commercial product, process, or service by trade name, trademark, manufacturer, or otherwise, does not necessarily constitute or imply its endorsement, recommendation, or favoring by the United States Government or any agency thereof or its contractors or subcontractors. The views and opinions of authors expressed herein do not necessarily state or reflect those of the United States Government or any agency thereof. 